\title{
CHARACTERIZATION OF EB-PVD YTTRIUM-STABILISED ZIRCONIA BY NANOINDENTATION
}

\author{
N. Vecchione ${ }^{1}$, K. Wasmer ${ }^{2}$, D.S. Balint ${ }^{1 \dagger}$ and K. Nikbin ${ }^{1}$ \\ ${ }^{1}$ Department of Mechanical Engineering, Imperial College London, London, SW7 2AZ, UK \\ ${ }^{2}$ Empa, Swiss Federal Laboratories for Material Testing and Research, Thun, 3602, Switzerland
}

\begin{abstract}
Nanoindentation was used to determine room temperature Young's modulus and hardness of the top surface and cross section of an electron beam physical vapor deposited yttrium-stabilized zirconia layer. In situ observations of indentations inside a scanning electron microscope (SEM) chamber were used along with normalized cross correlation (NCC) to evaluate the degree to which indentation of a columnar microstructure can be affected by elastic structural deformation. It was found that (i) the top surface Young's modulus is roughly twice that of the cross section. (ii) Upon isothermal heat treatment at $1100^{\circ} \mathrm{C}$ for 80 hours, an increase in Young's modulus and hardness occurred, to a greater degree in the bottom 20 $\mu \mathrm{m}$ of the cross section, and in the top surface, which was attributed to preferential sintering in these zones. (iii) NCC analysis of SEM observations of nanoindentation in the cross section gave an estimate of the extent to which elastic structural deformation is a constituent of the measured displacement. It was found that depending on the indenter location relative to the columnar microstructure, an underestimation of the Young's modulus by as much as a factor of four can occur.
\end{abstract}

Keywords: Nanoindentation; Thermal Barrier Coatings; Elastic Behavior; Multilayer Thin Films; Physical Vapor Deposition

\section{Introduction}

Thermal barrier coatings (TBCs) are widely used in gas turbines to protect superalloy blades and components from high operating temperatures that may routinely exceed the superalloy melting temperature (roughly $1200^{\circ} \mathrm{C}$ ). When combined with internal cooling, TBCs reduce actual component temperatures by as much as $200^{\circ} \mathrm{C}$. Their integrity is of critical importance; without protection, creep or other damage may occur. Typically the component life is that of its TBC..$^{\dagger}$

\footnotetext{
${ }^{\dagger}$ Corresponding author. Tel.: +44 (0) 207594 7084; email: d.balint@imperial.ac.uk
} 
A typical TBC is approximately $200 \mu \mathrm{m}$ thick and comprises 3 distinct thin layers (Fig. 1): (i) a metallic bond coat that also acts as an aluminum reservoir for oxide formation, (ii) a thermally grown oxide (TGO), usually alumina, which results from high temperature diffusion processes and protects the superalloy from corrosion, (iii) a low thermal conductivity ceramic, usually yttrium-stabilized zirconia (YSZ), which combined with internal cooling protects the superalloy from high temperature creep deformation and damage. The YSZ layer is typically deposited either by plasma spray or electron beam physical vapor deposition (EB-PVD); the latter method produces a columnar microstructure that makes the YSZ tolerant to in-plane strain and permeable to oxygen facilitating oxide layer formation. Strain tolerance reduces stresses in the YSZ resulting from thermal expansion mismatch with the much thicker superalloy substrate. The specific structure of EB-PVD zirconia involves 3 types of porosity [1]. The columns have tiny feather-like features, with associated nano-scale porosity, on their periphery inclined at a $50^{\circ}$ angle relative to the column axis. These features represent $1 / 2-1 / 3$ of the overall column width. The central part of each column has spherical pores. Finally, the columnar structure includes intercolumnar voids, a micro-scale porosity.

\section{Experimental procedure}

The objectives of this study were to characterize the effect of the columnar microstructure on apparent Young's modulus and hardness in EB-PVD YSZ by nanoindentation and make in-situ scanning electron microscope (SEM) observations of the indenter tip interacting with features of the columnar microstructure. Specimens tested originated from two $500 \mu \mathrm{m}$ square samples manufactured at Cranfield University [2] comprising a CMSX4 superalloy coated with either a Pt-aluminide or Pt-diffusion bond coat and EB-PVD YSZ ${ }^{1}$. Each of the original samples was quartered producing 2 batches of 4 specimens. Half of each batch underwent an isothermal heat treatment of 80 hours at $1100^{\circ} \mathrm{C}$ and the remainder did not undergo a heat treatment. One of the as-received and heat-treated specimens were selected from each batch and prepared for top-surface nanoindentation (TSN). The rest were prepared for cross-sectional nanoindentation (CSN). The TSN preparation consisted of coarse manual polishing with 1200 grit paper for approximately one minute to level the top surface. Prior to polishing, the CSN specimens intended for

\footnotetext{
${ }^{1}$ Any effect of the bond coat composition on the material properties of the YSZ is negligible.
} 
the MTS nanoindenter were mounted in Bakelite, with the remainder mounted in a custom-machined sample holder so that they could be removed from their mounting before use in the SEM nanoindenter. Cross sections were polished using five graded papers ranging from 200 to 2400 grit for 5 minutes at each grade. The 2400 grit paper corresponds to a median particle size of $0.75 \mu \mathrm{m}$, calculated by power law extrapolation of the relationship given in British Standard 8486-2 [3]. The roughness of the surface after polishing in fully dense areas is of the order of the paper particle size.

Nanoindentation experiments to measure material properties were performed using an MTS Nano Indenter XP with a diamond Berkovich tip having a radius of $0.2 \mu \mathrm{m}$. In all cases the loading rate was $1 \mathrm{mNs}^{-1}$. A total of 192 indents were made in the YSZ layer with a maximum load of $10 \mathrm{mN}$, with corresponding indentation depths no more than $500 \mathrm{~nm}$. To investigate the YSZ layer anisotropy, both cross-sectional (63 as-received, 64 heat-treated) and top surface (43 as-received, 22 heat-treated) indentations were carried out. Of the cross-sectional indents, 18 as-received and 21 heat-treated were in the bottom $20 \mu \mathrm{m}$ of the YSZ, nearest the TGO.

In order to observe the interaction between the indenter tip and the microstructure, several indentations were also carried out using a custom nanoindenter (although to depths of several $\mu \mathrm{m}$ ) and scratch tester enclosed in a SEM chamber allowing direct observation during indentation. Full details of the apparatus are given in [4]. A diamond cube corner tip having a radius of $0.2 \mu \mathrm{m}$ was used. The SEM indenter allowed simultaneous recording of the load-depth curve and a corresponding SEM movie.

\section{Data extraction and processing}

Values of Young's modulus and hardness were extracted from the indentation load-depth curves obtained with the MTS nanoindenter using the procedure developed by Oliver and Pharr [5],[6] for a homogeneous material. Results from both the MTS and SEM indenters were corrected to account for the effect of frame compliance. For materials of relatively high stiffness such as ceramics, loading frame compliance can have an appreciable effect on the final result. The stiffness of the MTS is roughly 1000 $\mathrm{mN} / \mu \mathrm{m}$. The custom SEM indenter is more compliant, mainly due to the load cell. Its frame stiffness was determined to be $263 \mathrm{mN} / \mu \mathrm{m}$ by comparing to MTS indentation curves from a previous study [7].

Nanoindentation was used intentionally to investigate the effect of microstructure on EB-PVD YSZ material properties; microindentation as in [8] was avoided in order to further probe features of the 
microstructure. It is assumed that the measured indentation response is effectively that of the homogenized volume local to the indenter tip. Therefore, fluctuations in elastic properties due to features of the microstructure can be detected on a scale larger than that of the local sampling volume by using an extraction method designed for a homogenous material.

The reduced modulus, $E_{r}$, calculated from the initial unloading slope of the load-depth curve, $S$, includes deformation of the indenter tip and is given by:

$$
E_{r}=\frac{\sqrt{\pi} S}{2 \beta \sqrt{A_{c}}},
$$

Where the correction factor $\beta=1.034$ is for a Berkovich tip, and where $A_{c}$ is the actual contact area calculated from the actual contact depth $h_{c}$ (which differs from the nominal machine value) [5],[6]. Given the plane strain Young's modulus of the indenter, $E_{i}{ }^{\prime}=E_{i} /\left(1-v_{i}{ }^{2}\right)$, that of the specimen (hereafter referred to simply as Young's modulus) is given by:

$$
E^{\prime}=\frac{E_{i}^{\prime} E_{r}}{E_{i}^{\prime}-E_{r}},
$$

and the hardness is defined as:

$$
H=\left(\frac{P}{A_{c}\left(h_{c}\right)}\right)_{\max } .
$$

As in previous experiments (e.g.[8]-[10]), measured values of Young's modulus for both crosssectional and top-surface indentation were scattered. The variability of the results presented here is attributed to discrete microstructure effects and subsidiary statistical scatter. A normal distribution was found to be inadequate in representing the extracted Young's modulus values due to scatter and the mode being considerably different from the mean. Therefore, mean and standard deviation were deemed inappropriate measures of the characteristic value and scatter in the data, respectively. Instead, a twoparameter Weibull distribution was assumed ${ }^{2}$ (see [10],[11]) with cumulative distribution function:

$$
F\left(E^{\prime}\right)=1-e^{-\left(\frac{E^{\prime}}{E_{w}^{\prime}}\right)^{m_{E}}},
$$

where $E^{\prime}{ }_{w}$ is the characteristic value of $E^{\prime}$ and $m_{E}$ correlates with the scatter in the data (larger $m_{E}$ indicates less scatter) [10]. The Weibull function is defined as (represented in a slightly different manner than usual, for transparency):

\footnotetext{
${ }^{2}$ The quality of this assumption is indicated by the degree of data linearity on a Weibull plot.
} 
$W\left(E^{\prime}\right)=\ln \left(\frac{1}{1-F\left(E^{\prime}\right)}\right)=\left(\frac{E^{\prime}}{E_{w}^{\prime}}\right)^{m_{E}}$

A Weibull plot consists of $\ln \left(W\left(E^{\prime}\right)\right)$ plotted against $\ln \left(E^{\prime}\right)$; the slope of a linear fit to data on a Weibull plot is $m_{E}$ and the characteristic value can be obtained from the property that $\ln \left(W\left(E_{w}^{\prime}{ }_{w}\right)=0\right.$.

\section{Results and discussion}

To obtain the actual cumulative distribution function, results were ordered by increasing value of $E^{\prime}$ and the corresponding ordered test rank $n$ was normalized by the total number of tests $N$ plus one:

$$
F\left(E^{\prime}\right)=\frac{n}{(N+1)} .
$$

Weibull plots for Young's modulus with linear fits to the data are shown in Figs. 2 and 3. The Weibull parameters corresponding to the fits are listed in Table 1, along with those for hardness. The anisotropic nature of EB-PVD YSZ is illustrated by Fig. 2 which shows Young's modulus values obtained in the cross section and top surface for both the as-received and heat-treated specimens. Very different zero crossings (characteristic values) are apparent for CSN and TSN in Fig. 2, suggesting a roughly transversely isotropic ${ }^{3}$ behavior consistent with expectations based on the columnar structure of EB-PVD YSZ.

For the TSN case, 11 values of $E$ were measured in excess of $230 \mathrm{GPa}$ (assuming $v=0.2$ in the extraction), regarded here as an absolute upper bound on $E$ based on the upper limit value for dense YSZ [10],[12] plus an estimated $10 \mathrm{GPa}$ bound on possible machine and extraction method errors. These values were deemed spurious and not included in the analysis, although it was verified that trends would be unaffected by their inclusion. We attribute the 11 spurious values to the indenter becoming slightly wedged between columns surrounding the tip during indentation which would affect the subsequent unloading slope. The top surface microstructure makes this far more likely to occur there than in the cross section. Indeed, such spurious values were not observed in the cross section.

Values of $E^{\prime}$ as small as approximately $\mathrm{e}^{2.73}$ were measured, or $E=15.33 \mathrm{GPa}$. As in [8], the smallest and largest values are presumed to correspond to indentations squarely on intercolumnar gaps and columns, respectively. A full range of intermediate values were measured between the two extremes.

\footnotetext{
${ }^{3}$ Although the characteristic stress-separation relation of intercolumnar gaps is likely different in tension and compression.
} 
Characteristic values $E_{w}^{\prime}$ for the as-received specimens were 81.16 and $151.88 \mathrm{GPa}$ for the cross section and top surface, respectively, and 88.11 and $205.99 \mathrm{GPa}$ for the heat treated specimens (see Table 1); the characteristic value of the effective in-plane Young's modulus is lower than in the direction perpendicular to the top surface by a ratio of roughly $1: 2$, for both the as-received and heat-treated specimens. This demonstrates the effect of strain tolerance of EB-PVD YSZ which is manifest as column separation under nanoindentation; in addition to material deformation local to the indenter tip, TSN and CSN engage the YSZ by elastic column compression and lateral bending, and elastic lateral and out-of-plane bending, respectively. A sketch of the effect of strain tolerance under indentation is shown in Fig. 4.

In thermal barrier coatings, the YSZ layer densifies preferentially with high-temperature exposure (sintering) as a result of diffusion processes. The results in Fig. 2 demonstrate the average of this effect with location, better quantified by the data in Table 1 . Complete closure of large gaps typically requires longer high temperature exposures, but average material properties can be affected sooner by fusion across smaller gaps and melding of the outer, featherlike structures of adjacent columns. Young's modulus and hardness increased with heat treatment consistent with [8],[13] although considerably more in the top surface when taking all indent locations into consideration. The gap area per unit area of top surface is greater than that of the cross section regardless of the dimensions ${ }^{4}$, thus amplifying the relative effect of sintering on the average behavior in the top surface. The reduction of scatter in the top surface results (increase in $m_{E}$ and $m_{H}$ ) also suggest sintering occurred there.

Fig. 3 reveals the extent of the through-thickness variations of Young's modulus and hardness in the cross section with thermal exposure. The vertical position of the indenter relative to the TGO was determined before each test using optical microscopy. The bottom region of the YSZ (about $20 \mu \mathrm{m}$, nearest the TGO) has an initially finer structure with smaller gaps (but more gap line length per unit area) than the rest of the cross section which has fully developed columns, and therefore should have different initial properties and sinter more readily. This was observed as indicated by the results summarized in Fig. 3 and Table 1. Young's modulus and hardness increased roughly by factors of 2 and 3, respectively, in the bottom $20 \mu \mathrm{m}$ as a result of 80 hours at $1100^{\circ} \mathrm{C}$, and much more than in the cross section as a

\footnotetext{
${ }^{4} \mathrm{Gap}$ area per unit area of top surface is $(2+\eta) /(1+\eta)$ times that of the cross section, where $\eta$ is the ratio of gap to column width.
} 
whole. In addition, the Weibull scatter parameters $m_{E}$ and $m_{H}$ increased considerably in the bottom $20 \mu \mathrm{m}$ suggesting a homogenization of the microstructure in that region.

In [13], the measured Young's modulus increased from 53.3 GPa in the as-received state to $104.1 \mathrm{GPa}$ after 100 hours at $1400^{\circ} \mathrm{C}$ for $\mathrm{CSN}$ and from $142.8 \mathrm{GPa}$ to $187.8 \mathrm{GPa}$ for TSN which is comparable to the results summarized in Table 1 for CSN and TSN in the bottom $20 \mu \mathrm{m}$ of the coating. The lack of a similar increase in Young's modulus with high temperature exposure for the cross section when all indents are considered (see Table 1) is believed to be a result of the randomness of the indenter position in this study; in Guo et al. [14] indent locations were intentionally chosen away from visible gaps and cracks. The lower values usually associated with gaps and cracks outside of the dense bottom zone are thus more likely to be avoided in [14].

The variation in the results obtained using the MTS nanoindenter can be largely attributed to the position of the indenter with respect to the microstructure. It is however suspected that elastic structural deformation of the columnar microstructure may in some cases be significant; it is important to dissociate material and structural deformation (e.g. column bending) if definitive quantitative conclusions are to be drawn as the latter is not accounted for in the extraction procedure of Oliver and Pharr [5],[6], leading to possible overestimation of the contact area and associated errors.

In order to investigate the degree of elastic structural deformation, indentations were carried out in an SEM chamber. Maximal final loads of $50 \mathrm{mN}$ and $100 \mathrm{mN}$ were used to ensure the contact area would not exceed the width of a column (at most, two columns were engaged), but be large enough that structural deformation could be easily observed in the SEM. In order to not obscure the view within the SEM, a cube corner tip was chosen since it has a much smaller total angle than a Berkovich tip; an additional consequence is that structural deformation will be less, thus a conservative lower bound on the effect of structural deformation is predicted.

The direction of observation was $70^{\circ}$ from the direction of indentation (see [4] for details). Video frames in chronological order of an indentation in the middle of a column and an intercolumnar gap are shown in Fig. 5, with corresponding load-depth curves in Fig. 6 (machine output given by solid symbols). The indentation perfectly centered on a column exhibited strikingly little inelastic deformation. Video showed that a chip was formed during the early stages of indentation as indicated in Fig. 5a, with a corresponding initial jump in displacement with almost no increase in load as shown in Fig. 6a; it was not 
included in the subsequent contact area calculation. It can also be observed from the video that there is some relative movement of the column to the right of the indenter, but that this is exclusively column displacement with no relative contribution from the indenter. This is attributed to the surface of the material being slightly inclined from $90^{\circ}$ relative to the indentation direction as a result of cutting and polishing. Indentation was accommodated by a combination of elastic and plastic material deformation local to the indenter tip and elastic column bending coupled to the surrounding intercolumnar gaps dilation and contraction. On the contrary, the indentation centered on a gap (Figs. 5b and 6b) involved little elastic structural deformation and appears more classical and in line with the situation considered to be the basis of [5],[6].

Without attempting to account for elastic structural deformation, the Young's modulus for the column indentation is $E^{\prime}=25.84 \mathrm{GPa}$ based on the procedure in [5][6] for a cube corner tip, and taking into account the effects of frame and indenter compliance. Normalized cross correlation (NCC) can be used on the SEM video frames to separate the combined effect of substrate movement (minimal, due to compliance of the fixation) and elastic column bending from the overall indentation depth response, thereby isolating the actual depth of indentation into the material. The load versus actual depth is plotted in Fig. 6a (open symbols), which shows that unloading closely follows the loading path which confirms there is little actual inelastic material deformation under the indenter and a large structural effect. The Young's modulus using this curve was found to be $E^{\prime}=104.13 \mathrm{GPa}$.

It was difficult to detect structural deformation in the gap indent of Figs. $5 \mathrm{~b}$ and $6 \mathrm{~b}$ due to the lack of NCC-trackable features, although it appeared to be minor. The Young's modulus extracted from the machine output was $49.13 \mathrm{GPa}$, roughly half the column value. Inspection of the video frames indicated that a change of slope in the load-depth curve (Fig. 6b) corresponded to the occurrence of a crack in one of the indented columns (Fig. 5b). The quantitative results in Fig. 6 for the indentation centered on a column do include a very slight underestimation of the contact area, and a corresponding slight underestimation in $E^{\prime}$ for that case, due to an apparent deviation from perfect planarity in the indented surface and hence the associated area function [5],[6]; in the case of indentation on a gap, the quantitative results are affected by the column fracture. However, these cases are qualitatively interesting because the values fit in the range obtained in the CSN distributions, indicating that such sources of error occur in 
classical batch indentation and are incorporated into the statistical scatter with ordinarily no correlation to indent location and local circumstances.

\section{Conclusions}

A set of indentation tests have been carried out on EB-PVD YSZ using a conventional MTS nanoindenter and a nanoindenter enclosed in an SEM chamber. The principal findings were: (i) Young's modulus measured in the top surface was approximately twice that in the cross section, (ii) sintering occurred with thermal exposure near the top surface and in the bottom $20 \mu \mathrm{m}$ where 80 hours at $1100^{\circ} \mathrm{C}$ increased the Young's modulus by roughly a factor of 2, (iii) significant elastic structural deformation may occur during cross-sectional nanoindentation leading to an underestimation of the Young's modulus as obtained by the Oliver and Pharr method, which can be corrected using NCC if SEM video frames of the indentation are available and trackable features can be identified. It was determined that the Young's modulus was underestimated by roughly a factor of four in the worst possible case (with respect to elastic structural deformation) of indentation in the middle of a column. The effect was observed to be of lesser magnitude when the indenter was located squarely on an intercolumnar gap.

Future studies will use FEA and analytical modeling correlated with SEM indents and systematic curve analysis in order to partition the work done by the indenter into local material deformation, elastic structural deformation and irreversible damage. Studies similar in principle have been carried out on other materials [15],[16], but without direct correlation between the load-depth response and actual events occurring during the indentation. This technique will allow more accurate material property identification for structured materials, and in the case of EB-PVD YSZ, identification of the intercolumnar gap stiffness which is needed to accurately model indentation and impact. Future studies will also include a more thorough examination of the influence of indent location relative to the TGO/bond coat interface, as well as the effect of different mounting materials. 


\section{References}

[1] T.J. Lu, C. Levi, H.N.G. Wadley, A.G. Evans, J. Am. Ceram. Soc. 84 (2001) 2937-2946.

[2] L. Chirivi, J. Nicholls, to be published (2008).

[3] British Standard ISO 8486-2 (2007).

[4] R. Rabe, J.M. Breguet, P. Schwaller, S. Stauss, F.J. Haug, J. Patscheider and J. Michler, Thin Solid Films 469 (2004) 206-213.

[5] W. Oliver, G. Pharr, J. Mater. Res. 7 (1992) 1564-1580.

[6] W. Oliver, G. Pharr, J. Mater. Res. 19 (2004) 3-20.

[7] B. Moser, K. Wasmer, L. Barbieri, J. Michler, J. Mater. Res. 22 (2007) 1004-1011.

[8] R. Wellman, H. Tourmente, S. Impey, J. Nicholls, Surf. Coat. Tech. 188-189 (2004) 79-84.

[9] Y. Gaillard, E. Jimenez-Pique, M. Anglada, Philos. Mag. 86 (2006) 5441-5451.

[10]E. Lugscheider, K. Bobzin, S. Barwulf, A. Etzkorn, Surf. Coat. Tech. 138 (2001) 9-13.

[11] C.K. Lin, C.C. Berndt, J. Mater. Sci. 30 (1995) 111-117.

[12] J. Thompson, T. Clyne, Acta. Mater. 49 (2001) 1565-1575.

[13] S. Guo, Y. Kagawa, Y., Ceram. Int. 32 (2006) 263-270.

[14] S. Guo, Y. Kagawa, Y., Ceram Surf. Coat. Tech. 182-1 (2004) 92-100.

[15] L. Zheng, A. Schmid, J. Lambropoulos, J Mater. Sci. 42 (2007) 191-198.

[16] K. Duan, R.W. Steinbrech, J. Eur. Ceram. Soc. 18 (1998) 87-93. 


\section{Tables}

Table 1

Weibull parameters for Young's modulus and hardness values extracted from the indentation data.

\begin{tabular}{lllllll}
\hline Orientation & Location & Thermal history & $\mathrm{E}_{\mathrm{w}}^{\prime}(\mathrm{GPa})$ & $\mathrm{m}_{\mathrm{E}}$ & $\mathrm{H}_{\mathrm{w}}(\mathrm{GPa})$ & $\mathrm{m}_{\mathrm{H}}$ \\
\hline Top surface & Random & As-received & 151.88 & 3.44 & 8.16 & 2.39 \\
& & Heat-treated & 205.99 & 4.29 & 15.99 & 1.91 \\
\hline Cross section & & As-received & 81.16 & 2.26 & 3.17 & 1.66 \\
& & Heat-treated & 88.11 & 1.93 & 5.87 & 1.69 \\
\cline { 3 - 7 } & \multirow{2}{*}{ Bottom $20 \mu \mathrm{m}$} & As-received & 65.51 & 1.93 & 2.71 & 1.40 \\
& & Heat-treated & 129.10 & 2.72 & 7.53 & 2.07 \\
\hline
\end{tabular}



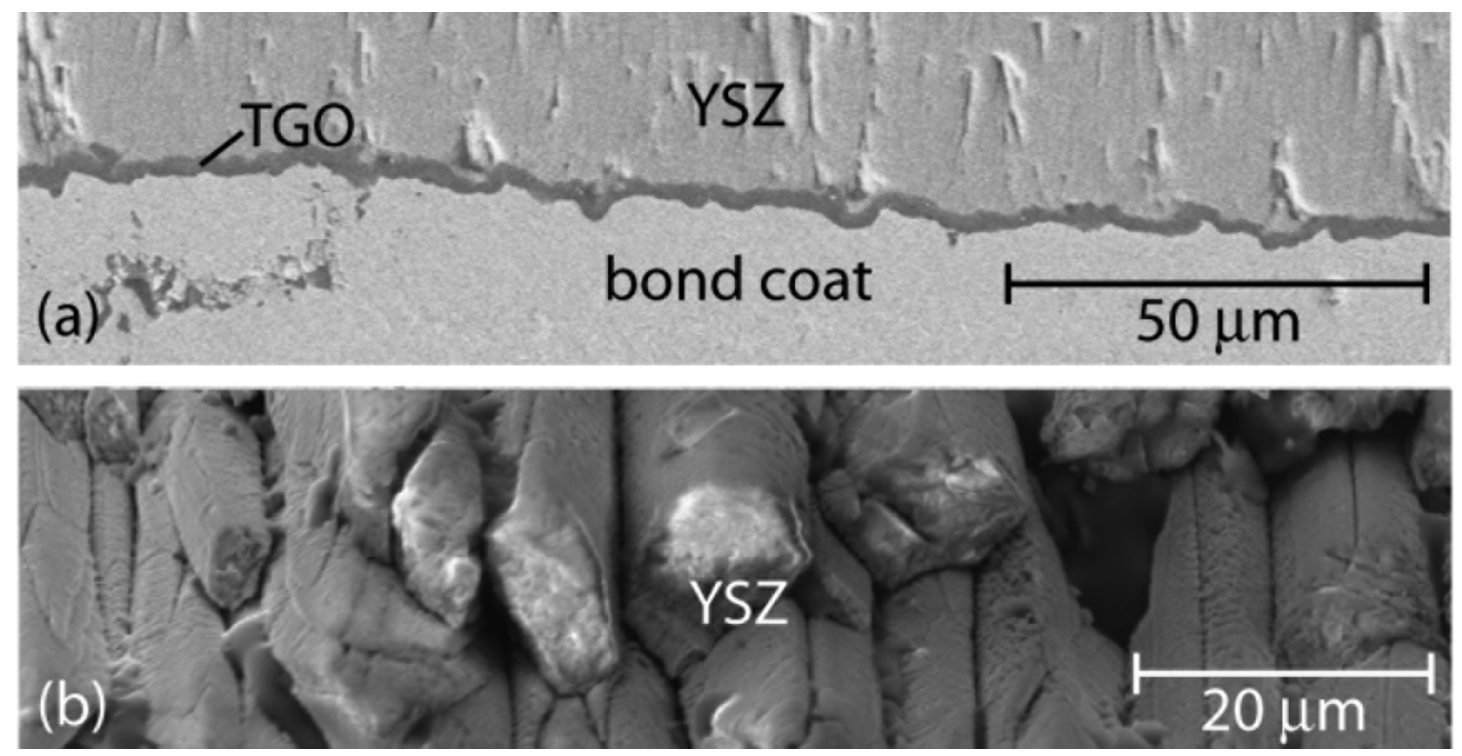

Fig. 1. (a) Polished cross section. (b) Oblique view of EB-PVD YSZ columnar structure.

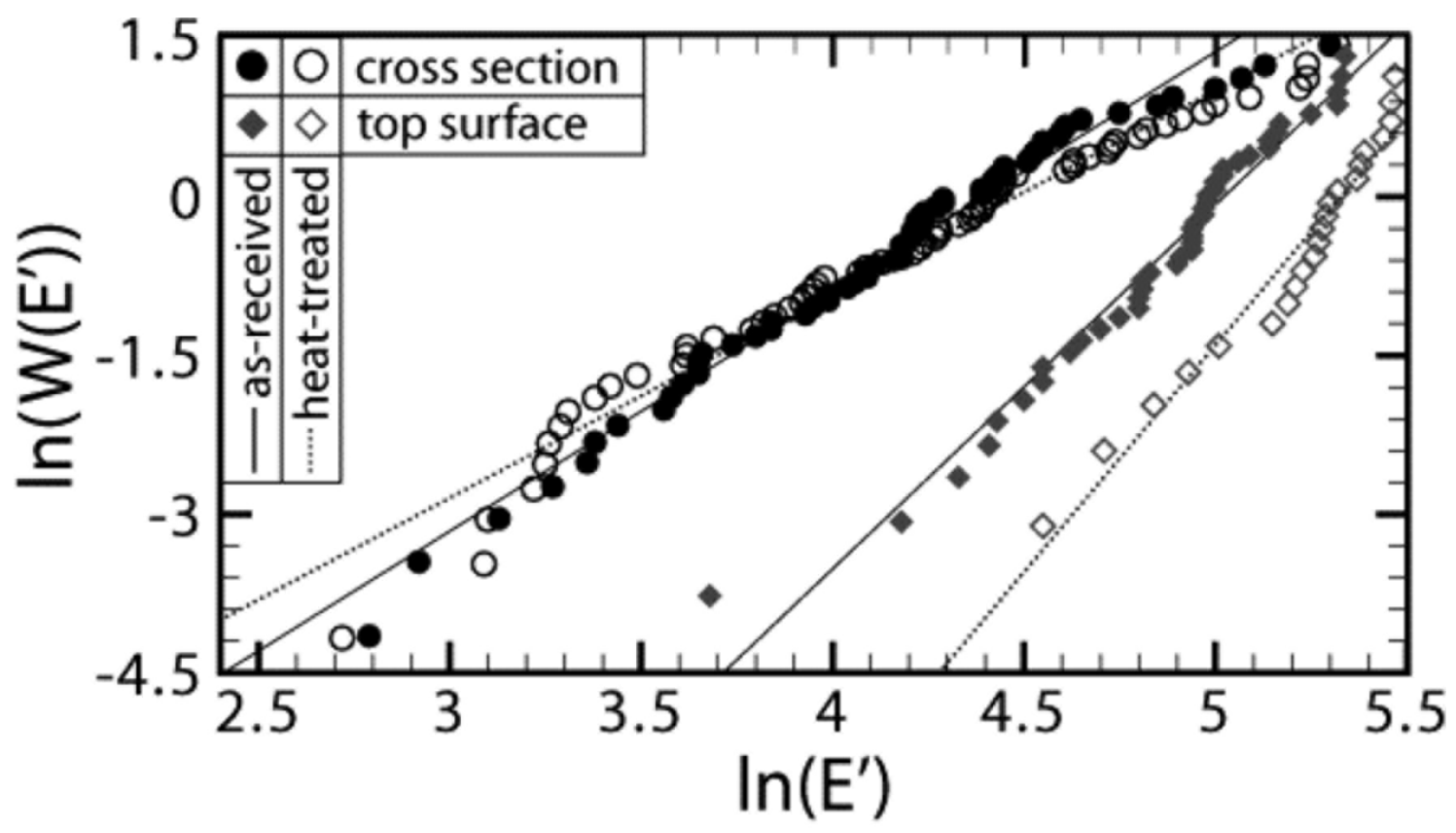

Fig. 2. Weibull plot of Young's modulus measured in the cross section and top surface. 


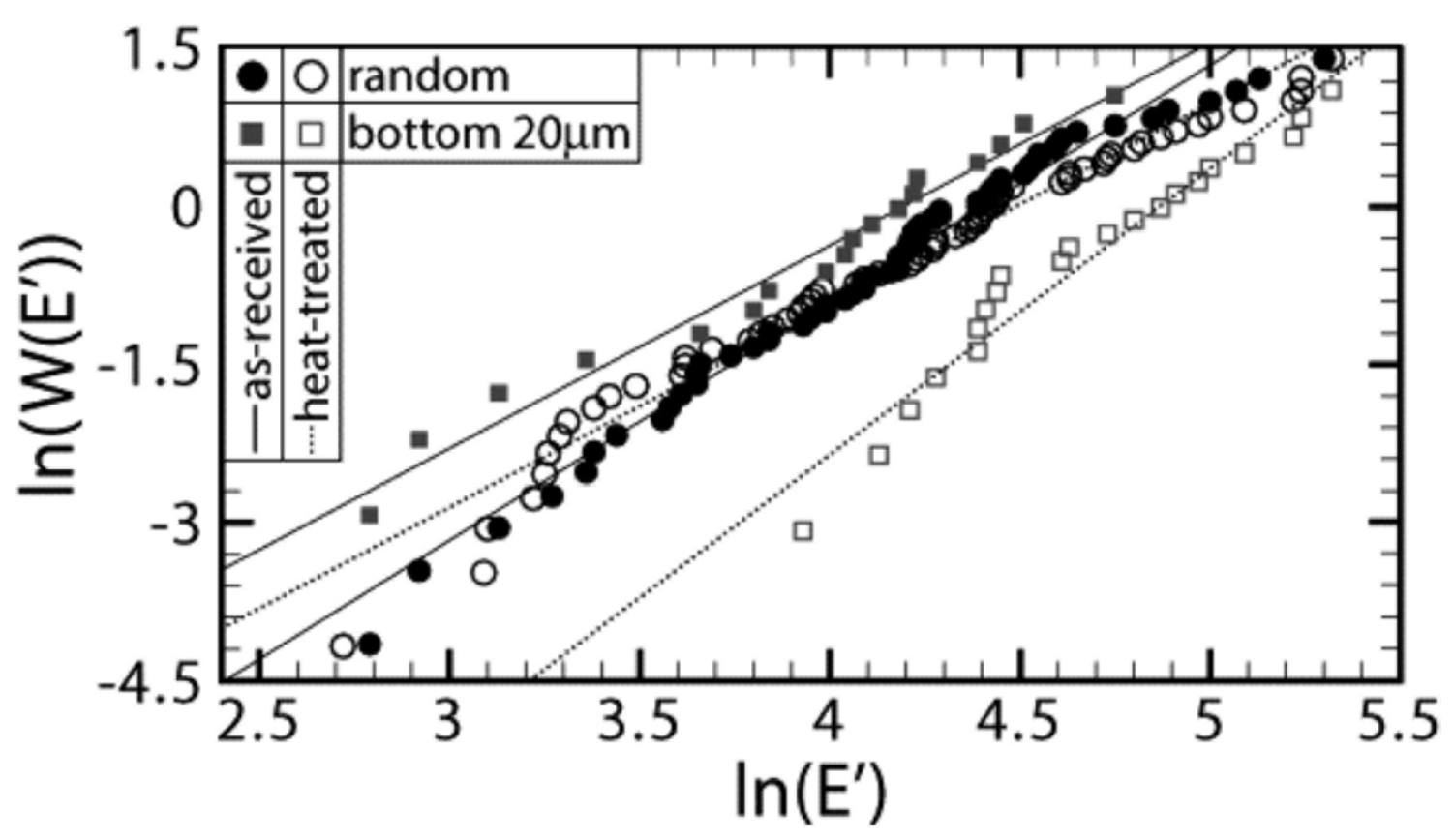

Fig. 3. Through-thickness variation of Young's modulus and the effect of heat treatment.

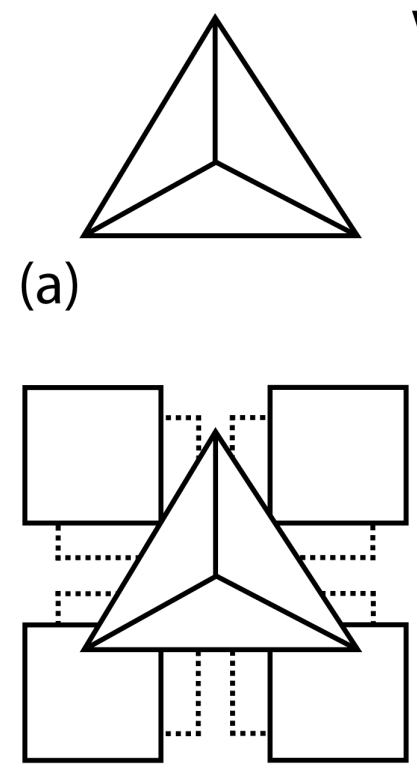

(b)

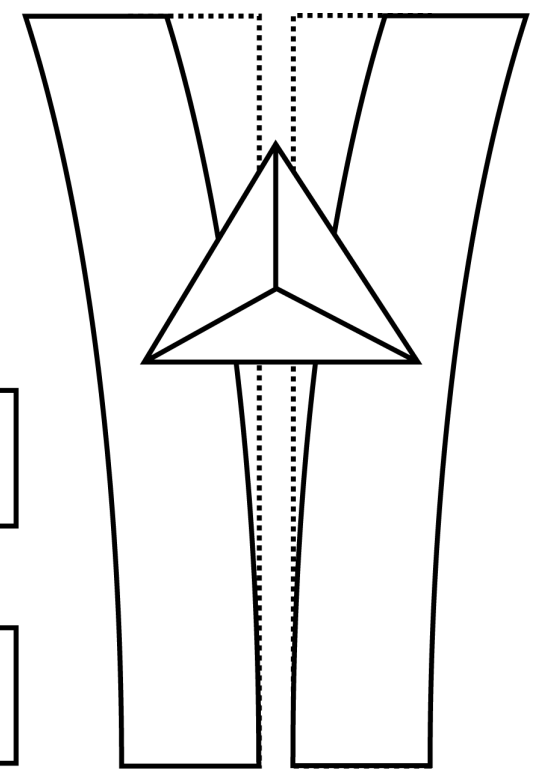

(c)

Fig. 4. Sketch of strain tolerance of EB-PVD YSZ under indentation. (a) Indenter tip. (b) Top surface. (c) Cross section. Dashed lines indicate original column positions. 

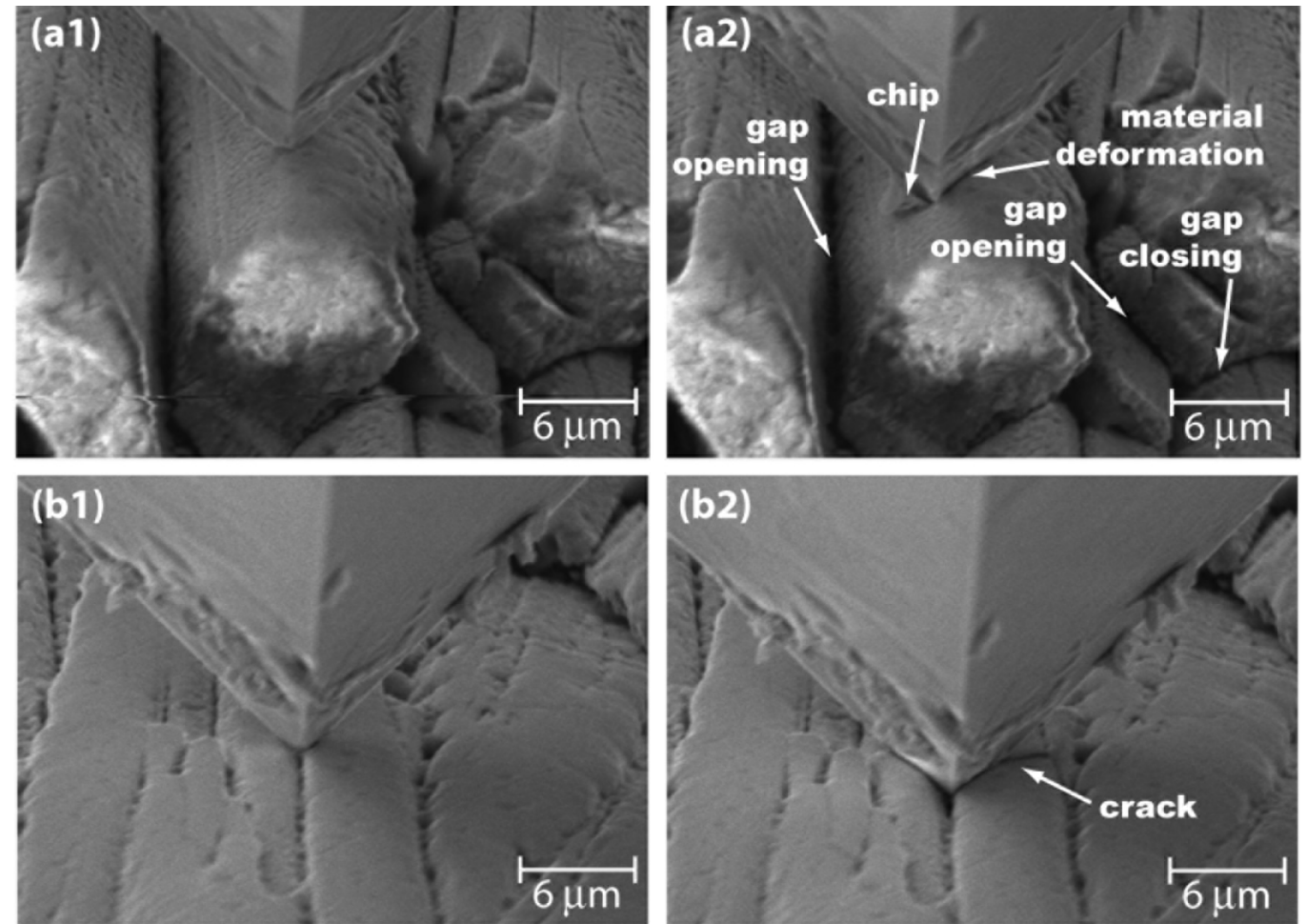

Fig. 5. Chronological in-situ SEM images of an indentation centered on a column $(\mathrm{a} 1, \mathrm{a} 2)$ and a gap (b1,b2).

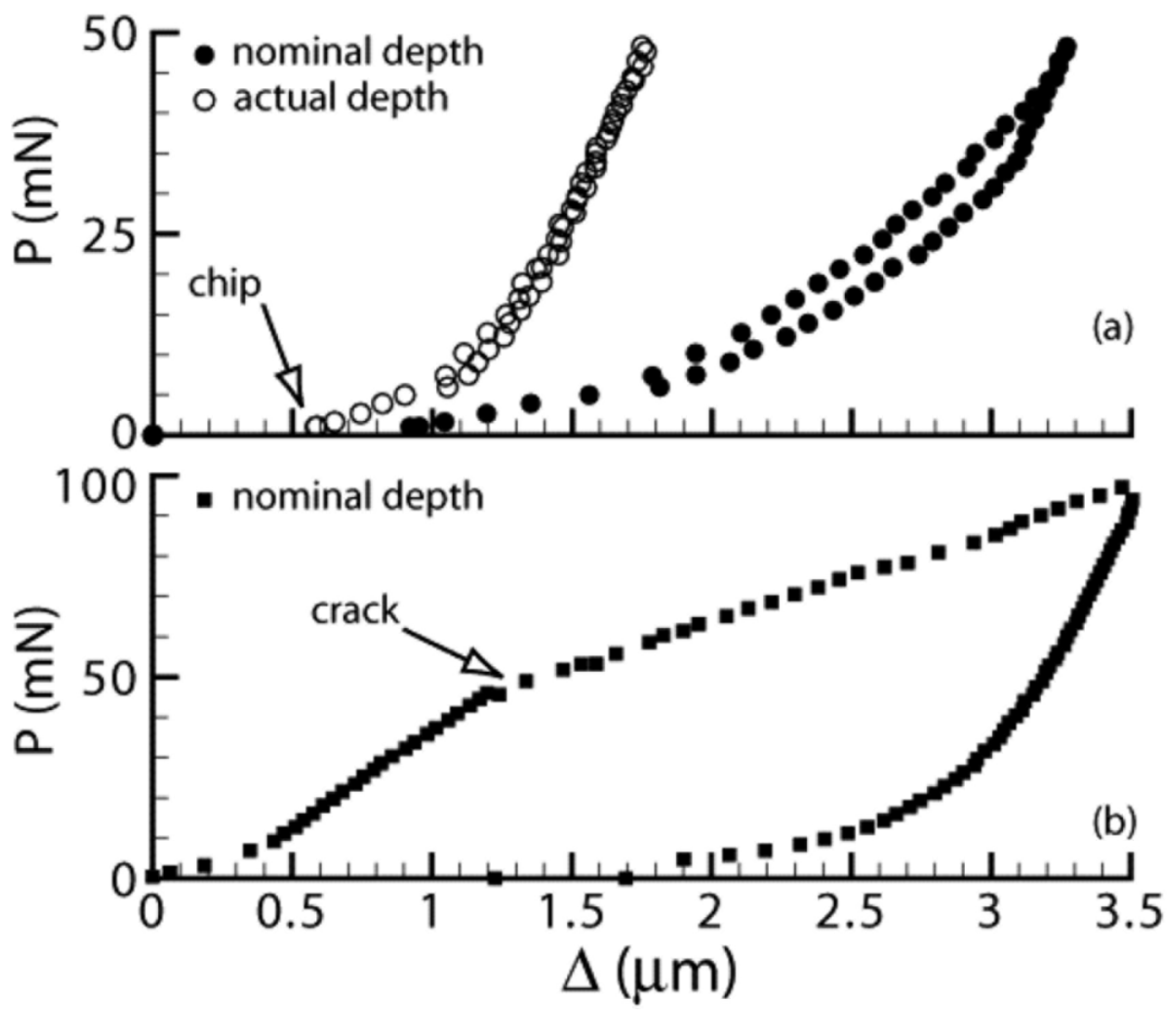

Fig. 6. Load versus indentation depth for indentation (a) centered on a column (b) gap (see Fig. 4). 